\title{
A Socio-Technical System Based on Gamification Towards Energy Savings
}

\author{
Piero Fraternali*, Francesca Cellina ${ }^{\dagger}$, Sergio Herrera*, Stelios Krinidis ${ }^{\ddagger}$, Chiara Pasini* $^{*}$, Andrea Emilio Rizzoli ${ }^{\dagger}$, \\ Cristina Rottondi ${ }^{\dagger}$ and Dimitrios Tzovaras $\ddagger$ \\ * Department of Electronics, Information, and Bioengineering, Politecnico di Milano, Italy \\ \{piero.fraternali, sergioluis.herrera, chiara.pasini\}@polimi.it \\ $\dagger$ Dalle Molle Institute for Artificial Intelligence (IDSIA) - University of Lugano (USI) - \\ University of Applied Science and Arts of Southern Switzerland (SUPSI) - \\ \{andrea.rizzoli, cristina.rottondi, francesca.cellina\}@supsi.ch \\ $\ddagger$ Information Technologies Institute, Centre for Research and Technology Hellas, Greece \\ \{krinidis, dimitrios.tzovaras\}@iti.gr
}

\begin{abstract}
This paper presents a gamification methodology and ICT solution currently under development in the enCOMPASS European project, which aims at implementing and validating an integrated socio-technical approach to behavioral change for energy saving. To this aim, innovative user-friendly digital tools are being developed to make energy data consumption available and understandable for different categories of users and stakeholders (household residents, office employees, school pupils, building managers, and utilities operators) and to enable their collaboration to achieve energy savings in efficient, costeffective and comfort-preserving ways. The enCOMPASS platform supports user-centered visualization of energy data from smart meters and sensors and uses the gathered information to provide its users with context-aware adaptive recommendations for energy savings. Moreover, it relies on gamification elements to increase effectiveness and produce greater and more durable behaviour change.
\end{abstract} tion.

Index Terms-Energy Saving; Behavioral Change; Gamifica-

\section{INTRODUCTION}

The world is looking for alternatives to traditional energy sources: fossil fuels have been linked to climate change [1], while at the same time nuclear energy sources still face issues related to safety and to the disposal of nuclear waste. Renewable energies are gaining momentum, but their supply is still limited and often subject to high volatility. All of the above lead us to consider that energy is still a limited resource and that ideas, methods, and solutions for energy efficiency and saving will continue to be needed in the future.

In the past years, the industry has been constantly and successfully working on energy efficiency, by producing devices and appliances that sensibly reduce their energy consumption; yet it can be observed the so called "rebound effect" [2], as exemplified by the case of LCD TVs: the consumption per square inch has been reduced in comparison with a traditional cathode-ray tube TV, but the size of the average TV has greatly increased, thus reducing the potential energy savings.

Actively engaging consumers to reduce their energy consumption, namely acting on the demand side, is therefore widely acknowledged as an urgent need for the future. For instance, recent research has shown that energy consumption feedback provided by in-home displays can lead to consumption reduction from $4 \%$ to $12 \%$ on average (with peak savings beyond 20\% [3]); however, this type of intervention can fail if not combined with measures that make consumers' behavioral change durable. Indeed, providing visual feedbacks on energy usage does not guarantee the achievement of consumption reductions by itself, as its success is largely dependent on user engagement [4]. Moreover, a careful design of visualization mechanisms should be adopted to make feedbacks readily understandable by the users and provide them with insights about their energy consumption habits and routines, possibly suggesting energy saving actions they could adopt [3].

From this perspective, the enCOMPASS project [5] aims at inducing a durable improvement in energy consumption in public and private buildings, by adopting a theoreticallyfounded multi-faceted approach for changing the behavior of consumers; the envisioned method integrates in novel ways gamified motivational stimuli and recommendations for energy saving, tailored to the user's context, activity, comfort level and phase in the behavioral change cycle. This objective requires understanding the user's typology and habits, her comfort level and current activity, to deliver timely, relevant, and personalized energy saving hints. To engage consumers, enCOMPASS develops an adaptive gamification platform aimed at dynamically motivating users to save energy. The platform processes smart meter and sensor data and collects a limited amount of profile data; it then estimates the users' consumption patterns and current activity context and provides them with personalized and context-aware recommendations of energy saving actions, supported by a rewarding system that is designed to foster the level of engagement (i.e., the extent to which recommendations are put into practice) and the consequent behavioural change and energy saving. In particular, the enCOMPASS platform tracks users' activity related to energy consumption (captured with smart meters), personal attitudes (identified through surveys and application activity logs) and context (estimated from comfort and sensor readings), and, by means of a recommendation engine, maps such an information 
into energy saving tips tailored to their own profile and current status. Effectiveness of the enCOMPASS platform will be assessed in real-life field trials, involving households, schools and public buildings in three European pilot sites (Germany, Greece and Switzerland).

In this paper we focus on the description of the enCOMPASS gamified platform. After a brief overview of the related literature (Section II), Section III presents the overall enCOMPASS architecture and Section IV details the main components of the gamified platform. Section V outlines the design of the validation trials and the methodologies to assess its impact.

\section{RELATED WORK}

The deployment of smart meters in an increasing number of households has boosted research on methodologies for inducing behavioural change based on energy consumption feedback. Several energy saving applications embedded in users' everyday environment have been proposed [6], [7], using visualized consumption feedback and gamified social interactions to motivate people to adopt energy-efficient lifestyles. Several are data-oriented (e.g. bar or pie charts of consumption [8], [9]), some are closely connected to the real consumption contexts (e.g. floor plans [8]), others are metaphorical (e.g. traffic lights and gauges [8]), playful and ambient (BeAware [10] and SmartH2O [11]), or connected to nature or animal habitats (eco-visualization [12], [13]).

Gamification, in particular, has become a popular approach to behavioural change for resource-efficiency projects as a motivational tool, as it encourages users to set saving goals to achieve virtual prizes like badges and medals. Examples of such approach can be observed in the projects Makahiki [14] and LEY [15]. It has also been used to provide information and training to users towards resource-efficient behaviours (projects like Power House [16] and the "Water Mansion" game [17]) or to promote social collaboration and competition for resource saving (Wattsup [18], Social Power [19], [20] and Social Electricity [21]). Initially targeting households, gamified approaches have recently started been applied also to public buildings (GreenSoul [22] and ChArGED [23]) and schools as well, such as in the GAIA project [24].

We especially draw inspiration from the SmartH2O project, which aims at raising awareness of water consumption and at encouraging efficient water-use behaviours [25], by means of a gamified platform providing consumption feedback with a visualization interface. Users are provided with water saving tips and learning elements (videos), encouraged in competing for the highest water savings using a points-goals based competition and in getting social recognition through virtual prizes badges and leaderboards, and enabled to share their platform activities on popular social networks [11].

In enCOMPASS we adopt a similar approach, extending it in several aspects in order to overcome key limitations related to the provision of static, one-size-fits-all tips and recommendations for change [26]. In fact, SmartH2O offers its users a fixed set of tips and educational videos, which may lose effectiveness in case they are not related with the user specific action or context [27], or the time of the action [28]. Instead, enCOMPASS incorporates a dynamic recommendation engine that considers the users' profiles and the context of their actions (by means of data on movement, temperature, humidity and luminance automatically gathered by sensors), that allow to provide users with timely and personalized recommendations. Besides just collecting additional information by sensors and providing suggestions, in presence of automated home management solutions already deployed at the customers' premises, enCOMPASS is also able to automatically perform energy saving actions, when specific situations are detected. For example, if the enCOMPASS platform understands that in an empty room (no motion detected by movement sensors) a light is on (electricity consumption detected by smart meter data and energy consumption disaggregation algorithms or luminance sensors), it automatically turns the light off.

Following an initial idea already developed by SmartH2O, to further encourage user awareness and energy saving actions, enCOMPASS does not limit to a digital gamified platform which offers virtual rewards, but it is backed up by a tangible, real-life matching energy-related card game, named Funergy. The board game is offered to enCOMPASS users as a reward in return for their energy efficient behaviour or for high levels of activity with the enCOMPASS platform. It is designed to be played as a common card game in school classrooms or family contexts but users get the most by exploiting its digital extension which connects it to the activities performed on the digital enCOMPASS platform. Using Funergy therefore generates additional rewards, in a reinforcing virtuous circle.

By bringing these elements together, enCOMPASS aims at influencing users to adopt long-lasting energy efficient behaviours, in order to produce higher energy consumption reduction rates with respect to related projects.

\section{OVERVIEW OF THE ENCOMPASS ARCHITECTURE}

The original concept of the enCOMPASS architecture integrates several components and processes aimed at triggering individual and collective behavioural response to specific energy conservation policies. The framework collects data generated by electricity meters and sensors installed at the users' premises, as well as data derived from users actions on a gamified application. The collected data are processed using data analysis instruments. The sensor data stream is exploited by an activity tracker subsystem, which infers the current activity of the user in the building; the consumption data stream is exploited by a disaggregator, which estimates the partition of the total metered consumption into end-uses (e.g., water heating, specific appliances, etc); both the sensor and consumption data stream are fed to a recommender subsystem, which computes in-context adaptive recommendations, tailored to the user's current context and activity. The data analytics processes also support users and building modeling, which helps the administrator of an utility company to monitor, simulate, and ultimately better manage the demand.

Besides data analytics, the input data streams are also used as a device to enhance awareness: measured statistics on consumption are exposed to the users via a consumer app comprising gamification elements that promote behavioural changes by 
means of game-like elements, such as points, achievements, and rewards, related both to measured energy savings and to the user's response to the delivered recommendations that require her to act on the building (e.g., changing the set point of the heating system). The consumer app also rewards the user for entering a few psychographic variables, e.g.,the composition of the family or the presence of appliances, which improve the accuracy of the recommender system, of the user and building modeler and of the disaggretator.

\section{COMPONENTS OF THE ENCOMPASS PLATFORM}

The goal of the enCOMPASS platform is to transform the data streams acquired from smart ambient and electricity consumption sensors into representations intelligible by the consumers and into recommendations of energy saving actions based on the current context and activity of the users. This task is accomplished by the coordinated cooperation of multiple components, embedded in the technical architecture portrayed in Figure 1.

a) Sensors data acquisition: this component ingests energy consumption data from smart electricity meters and smart plugs at individual appliance level, and sensor data (presence, temperature, luminance, humidity, etc.). It acquires data from multiple heterogeneous metering infrastructures, home control systems, and sensing equipment, converts them to a centralized uniform data model and serves the integrated data to all the other components of the platform.

b) User data acquisition: it handles the acquisition of user-related psychographic variables from multiple sources: profile information gathered with a gamified mobile apps (e.g., about household composition and existing appliances), results of instant polls (e.g., quick feedback provided via app on activity or comfort conditions), along with data inferred in a privacy preserving way, through activity tracking measurements produced by sensors (e.g. user presence in a room).

c) Data analysis and user modelling: this component includes algorithms for extracting activity data from sensor measurements and app data, profiling different types of user behaviour, inferring activity context, predicting reactions to stimuli (e.g., energy saving tips). More in detail, the engine takes as input the raw data from the sensors, i.e. the indoor environmental conditions, and fine grained energy consumption measurements from the smart meters. All these data are processed utilizing advanced analysis and classification techniques (such as those described in [29], [30]) for deducing the number of occupants and/or presence/absence in a building and categorizing the comfort levels of the building occupants. The comfort is divided into two main classes, i.e., visual and thermal comfort. The visual comfort is referred to the human perception of the luminance in a building. the thermal comfort is instead related to the human perception of the indoor temperature. The thermal comfort is based on the Mean Predicted Values (PMV) [31], whereas the visual comfort is based on the Kruithof diagram [32]. The component also disaggregates the raw information of the total energy consumption into end uses, thus inferring the electrical appliances in use and, consequently, the activities in a building, (e.g. cooking, resting, sleeping, etc.) -. All the extracted values are stored in the database along with the reference timestamp, for further use by the recommendation engine and other enCOMPASS components.

d) Data analysis and building modeling: this component implements algorithms for estimating the comfort levels and energy behaviour of buildings, based on a reduced set of measured input parameters dependent on the class of the buildings. It allows for the estimation of the impact of consumers actions on energy consumption and on the status of the building, so to help the recommender component to prioritize energy saving recommendations based on both the user's context and activity and on the potential impact of the suggested action. The base model includes: thermal building characteristics (i.e., envelope materials, building size), user profile, local climate data, energy consumption of subsystems (i.e., HVAC, plug loads etc.).

e) Adaptive in-context action recommendation: this component embodies the core intelligence of the system, which computes and delivers actionable energy saving suggestions from all the different types of data available, including consumption and sensor data, user profile data, building information and user actions in the consumer app and in the building control system. The recommender cluster users in categories and exploits their activity patterns to elaborate energy saving recommendations for a specific user category and context. A rule-based engine maps consumption and activity patterns into energy saving tips, which are delivered to users as notifications in the consumer app. User's feedback on the suitability and utility of recommendations is also collected by the consumers app and fed back to the component, which exploits such information to adjust the recommendation rules. To overcome the cold-start problem, the recommender is endowed with generic rules, which apply to all classes of users and buildings, which are used to initialize the feedback loop between the recommender and the user.

f) Behavioural change consumer apps: these web and mobile applications are developed to help users make sense of their energy usage and engage in energy saving efforts, supported by gamification techniques. They are also responsible of delivering to the users the personalized suggestions elaborated by the recommender, in the form of notifications. Their interface comprises several sections, targeting different phases of the behavioral change process and including: the visualization of consumption and comfort data over time, charted in histograms; the alternative representation of consumption data with visual metaphors that convey the impact of energy saving under different perspectives, e.g., emphasizing the monetary or ecological value of the achieved savings; the collection of generic and personalized energy saving tips, which help nudge the user from the intention of saving into concrete practice; a goal-setting area, where users can reinforce their willingness of acting by setting individual or collective energy saving goals; a profile data input area, where users can gain gamification points by inputting information on their household and building; and a gamification area, where users can inspect the complete history of their actions and achievements, the received and available rewards, and 


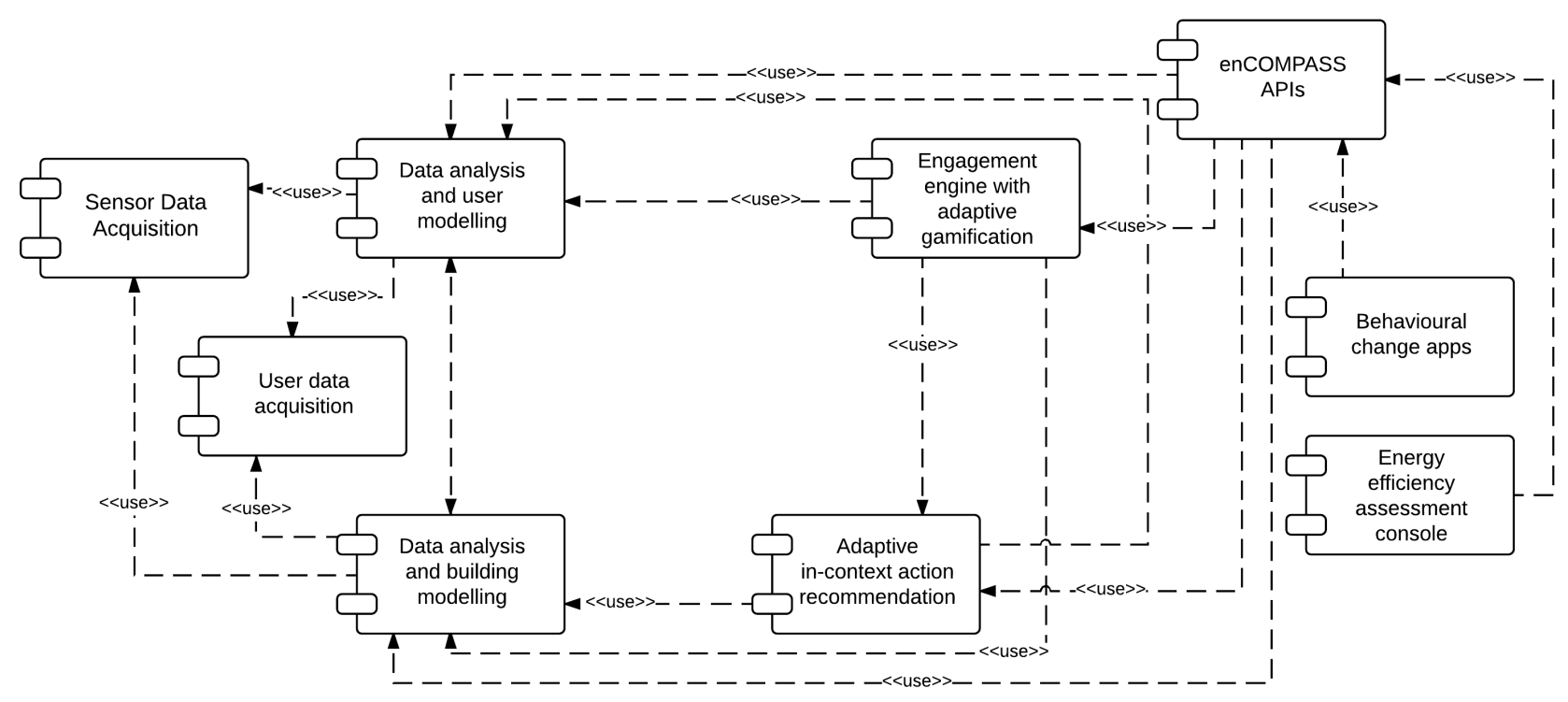

Fig. 1. The components of the enCOMPASS platform

compare their results with those of their peers. The app feeds back user's achievements to the consumers' community in the form of individual or team leaderboards, which are designed with different time horizons (e.g., weekly and over the entire experimentation period) to support both early adopters with a long history of interaction with the system and newcomers.

g) Engagement Engine with adaptive gamification: This back-end component supports the gamification mechanisms embedded in the consumer app by recording the actions of the users in the consumer app and transforming them into a variety of achievements and rewards, computed by means of a rule-based mapping mechanism. Actions are abstract occurrences that can be associated to a variety of sources, such as data from smart meters and building sensors (e.g. saving a set amount of energy), and users themselves. The mapped actions include: shift in the consumption patterns, achievement of reduction goals, input of user or building profile data, response to polls, invitation of people to join the activities, etc. Rewards can be immaterial (points, badges) or material (coupons, redeemable goods or the Funergy game mentioned above). Availability of achievements and rewards allows energy utility companies to challenge their users with energy saving goals to be achieved each month. The maximization of the impact on energy consumption behavior is pursued by making the gamification model flexible and adaptive, taking into account the users progress along the behavioral change cycle, as defined in [33]. Multiple incentives and feedbacks are computed and displayed in different areas of the consumer app, so to adapt the behaviour change stimuli to the context-of-use and progress status of the user; individual and collaborative consumption goals, user progress in saving goal completion, user profile-dependent reward rules, users location and activity, indoor climate parameters, and interaction history are used as inputs to compute the achievements of users and transform them into points in the gamification exercise.

h) Digital Game Extension: Besides in-app gamification, enCOMPASS supports energy saving awareness also via traditional gaming, implemented with a hybrid game, called Funergy, mixing real and digital play. The digital part implements an Energy Trivia Game, which complements the game play of the Funergy real card game; Funergy is designed with the purpose of increasing the awareness of users, especially kids, about the importance of energy efficiency labels in domestic appliances. The digital game is implemented as a web and mobile app, which pulls from a dedicated backend server the content and play statistics necessary to populate its user interface (e.g., trivia quizzes, difficulty levels, correct responses, points, players skill level and achievements, etc.); it targets both casual players and users of the enCOMPASS gamified platform and of the Funergy card game; the latter can use the digital game as a side-kick to overcome critical situations in the real card game.

i) Energy efficiency assessment console: it is a web and mobile app whereby building managers (BMs) and utilities can analyse aggregated consumption and sensor data of all the users and set saving goals/incentives. It allows BMs to create customized dashboards to get different perspectives about energy consumption in one or more buildings, such as the current status of one building or user, the cumulative consumption over a period, or the comparison of multiple users and/or buildings, etc. The console retrieves consumption and sensor data stored in the platform database and displays them using multiple visualization widgets (e.g. plots, bars, pies, maps, donuts, bubbles, polar and spider charts, etc.), allowing the BM to select the ones that most fits his/her needs. Widgets can be parameterized to display specific data for an arbitrarily chosen period.

j) enCOMPASS APIs: they provide on-demand, cloudbased web services that enable end-user applications to access and exploit the enCOMPASS processes. The data and service APIs enable developers to access both enCOMPASS functionalities and data while implementing their own services.

\section{DESIGN OF THE ENCOMPASS REAL-LIFE TRIAL}

The enCOMPASS project has been launched in November 2016 and it is now approaching a half of its lifetime. The 
first year of the project was devoted to the collection of the user requirements, to the design of the initial architecture of the enCOMPASS platform and to planning field trials with real-life end users. Three behavioural change apps are currently being developed: one for households, one for schools and one for public buildings. The app for households targets domestic users in a family group, the app for schools targets students in classrooms groups and the app for public buildings targets colleagues working in the same building, grouped based on their office, and visitors of the public areas of the building. Effectiveness of these apps will be assessed by field trials in three pilot sites (Switzerland, Germany and Greece), in each of whom one hundred households, one school and one public building are involved. Overall, around two thousand users will be involved in the field test (intervention group). Households are currently being selected in all sites: participation is voluntary and it is favored by public communication campaigns aimed at recruiting interested citizens, prizes offered by the utility companies involved in the project and direct invitation letters sent to a sample of around two hundred households per site (representative sample respect to the total population of each utility company, stratified based on type of household, type of building and type of heating system). Schools and public buildings have instead already been selected, by direct agreement with the Municipalities and main public institutions of each test site. Interaction with schools was particularly appreciated, since it will go beyond just using the virtual enCOMPASS behavioural change apps, thanks to the Funergy board game, organization of specific classes focusing on energy saving and energy efficiency topics and creation of synergies with the usual curricula on energy and the environment.

All the buildings participating in the trial will be endowed with the enCOMPASS platform (smart meters for electricity consumption, sensors for motion and indoor and outdoor temperature and humidity, smart plugs and behavioural change app) and energy-related behaviour of its users will be monitored over time. In particular, smart meters have been on purpose installed in the Swiss site, while they were already available in Germany and Greece. Sensors, instead, will be installed on purpose for the trial: households will install them in the living room, schools in each classroom and in the foyer area, public buildings in each office, in the common areas and in visitors areas. In the same areas, for school and public buildings a tablet will be installed, in order to let groups of students/colleagues/visitors frequently interact with the enCOMPASS platform.

Both electricity consumption and smart meters data will be continuously gathered, with a frequency of fifteen minutes. Besides them, the field trial will also collect user perceptions and attitude data, as proxies for behavioural predictors. Such data will be gathered by using methods typically employed in user-centered design and psychological research, such as questionnaires, focus groups and interviews. Impact of the enCOMPASS platform will be measured by comparing the set of data collected during the enCOMPASS intervention period, with respect to the same set of data collected before the intervention. To univocally attribute differences in consumption and behavioural predictors to the enCOMPASS platform, for households the trial also envisions a control group in each pilot site, that is a group of users that are similar to those of the intervention group, and are monitored in order to obtain data uninfluenced by the enCOMPASS platform - in fact they are not treated by the enCOMPASS tools. Also for them, data will be collected before and after the intervention. Therefore, comparing differences in the set of data collected from the intervention group and the control group, will allow for and assessment of the net effect of the enCOMPASS platform. Conversely, no control group is envisioned for schools and public buildings, due to the difficulty of individuating buildings with similar characteristics (in building type, pupil/employee composition etc.) in the same geographical and climate context.

Three releases of the enCOMPASS platform are actually envisioned: the first one (R1) in April 2018, the second one (R2) in November 2018, the third one (R3) in October 2019, at the end of the project. Release R 2 will offer more advanced functionalities of the enCOMPASS platform, while release R3 will simply include bug fixes and final refinements, as identified from the trial themselves, and therefore does not need to be tested. Therefore, three monitoring periods are scheduled: (i) before the intervention electricity consumption baseline data are collected (one year, from May 2017 to April 2018), (ii) during "intervention 1" (use of R1, May - October 2018) and (iii) during "intervention 2 " (use of R2, November 2018 - July 2019). Note that slightly different periods are set for schools, in order to take account of the Summer holidays.

The three monitoring periods are run consecutively and, during these periods, meters and sensors data of the involved users (both intervention and control groups) will be continuously collected. A three-wave survey (one survey for each monitoring period) will instead gather behavioural data on attitudes and perceptions. The assessment of the impact on electricity consumption data will be performed by means of the eeMeasure methodology [34], which allows for direct comparison with other energy saving projects and interventions developed throughout Europe, and fits to trials both with and without a control group. Assessment of the impact on behavioral data will instead be performed by comparing Likert-scale answers to the same questions across the three waves of the survey, and by accounting for their variations. The target KPI values are $20 \%-25 \%$ reduction in average weekly electricity consumption and 1 point increase on a 5-points Likert scale $(+20 \%)$ in the user awareness of energy consumption, knowledge of energy saving actions and intention to save energy.

\section{CONCLUSION}

This paper describes the ongoing research of the enCOMPASS project, which aims at investigating the impact of ICTbased behavioral change tools for energy savings. Provided that the project team will manage to retain participants' attention and prevent high drop-out rates, the enCOMPASS real-life trial will enable a deeper understanding of what works and what does not in the design and deployment of an original mix of instruments, including contextual, personalized energy 
saving recommendation, delivered with web and mobile user interfaces, gamification-based stimuli, such as points, achievements and rewards, and original game concepts, mixing real and digital games to increase awareness and promote education towards sustainable energy consumption.

\section{ACKNOWLEDGEMENTS}

This work is partially supported by the "enCOMPASS Collaborative Recommendations and Adaptive Control for Personalised Energy Saving" project funded by the EU H2020 Programme, grant agreement no. 723059.

\section{REFERENCES}

[1] S. Manabe and R. T. Wetherald, "Thermal equilibrium of the atmosphere with a given distribution of relative humidity," Journal of the Atmospheric Sciences, vol. 24, no. 3, pp. 241-259, 1967. [Online]. Available: http://dx.doi.org/10.1175/1520-0469(1967) 024〈0241:TEOTAW $\rangle$ 2.0.CO;2

[2] V. Tiefenbeck, V. Tasic, S. Schöb, and T. Staake, "Long-lasting effects or short-term spark? on the persistence of behaviour change induced by real-time feedback on resource consumption." in ECIS, 2016, p. ResearchPaper84.

[3] K. Buchanan, R. Russo, and B. Anderson, "The question of energy reduction: The problem(s) with feedback," Energy Policy, vol. 77, pp. 89 - 96, 2015. [Online]. Available: https://www.sciencedirect.com/ science/article/pii/S0301421514006739

[4] T. Hargreaves, M. Nye, and J. Burgess, "Keeping energy visible? exploring how householders interact with feedback from smart energy monitors in the longer term," Energy policy, vol. 52, pp. 126-134, 2013.

[5] P. Fraternali, S. Herrera, J. Novak, M. Melenhorst, D. Tzovaras, S. Krinidis, A. E. Rizzoli, C. Rottondi, and F. Cellina, "enCOMPASS - an integrative approach to behavioural change for energy saving," in Global Internet of Things Summit (GIoTS), 2017. IEEE, 2017, pp. 1-6.

[6] A. Barbato, C. Bolchini, A. Geronazzo, E. Quintarelli, A. Palamarciuc, A. Pit, C. Rottondi, and G. Verticale, "Energy optimization and management of demand response interactions in a smart campus," Energies, vol. 9 , no. 6,2016 .

[7] C. Rottondi, M. Duchon, D. Koss, A. Palamarciuc, A. Piti', G. Verticale, and B. Schaetz, "An energy management service for the smart office," Energies, vol. 8, no. 10, pp. 11667-11684, 2015.

[8] P. Monigatti, M. Apperley, and B. Rogers, "Power and energy visualization for the micro-management of household electricity consumption," in Proc. Conf. Advanced Visual Interfaces. ACM, 2010, pp. 325-328.

[9] T. Schwartz, G. Stevens, T. Jakobi, S. Denef, L. Ramirez, V. Wulf, and D. Randall, "What people do with consumption feedback: A long-term living lab study of a home energy management system," Interacting with Computers, vol. 27, no. 6, pp. 551-576, 2014.

[10] "Be aware project - boosting energy awareness with adaptive real-time environments," EU FP7-ICT, No. 224557,, 2008-11, accessed: 25 February 2017. [Online]. Available: http://www.energyawareness.eu/

[11] P. Fraternali, L. Caldararu, J. Novak, C. Pasini, G. Baroffio, M. Tagliasacchi, and A. E. Rizzoli, "Smarth2o d.6.2: Platform architecture and design," 2015, accessed: 10 May 2017. [Online]. Available: http://smarth2o.deib.polimi.it/wp-content/uploads/2015/09/ sh2o_D6.2_POLIMI_WP6_platform_architecture_and_design_v3.2.pdf

[12] A. Gustafsson, M. Bång, and M. Svahn, "Power explorer: a casual game style for encouraging long term behavior change among teenagers," in Proc. Adv. in Comp. Entertainment Tech. ACM, 2009, pp. 182-189.

[13] A. Gustafsson, C. Katzeff, and M. Bang, "Evaluation of a pervasive game for domestic energy engagement among teenagers," Computers in Entertainment (CIE), vol. 7, no. 4, p. 54, 2009.

[14] G. Lee, Y. Xu, R. S. Brewer, and P. M. Johnson, "Makahiki: An open source game engine for energy education and conservation," Foundations of Digital Game, 2013.

[15] R. N. Madeira, A. Vieira, and N. Correia, "Personalization of an energy awareness pervasive game," in Proceedings of the 2012 ACM Conference on Ubiquitous Computing. ACM, 2012, pp. 619-620.

[16] B. Reeves, J. J. Cummings, J. K. Scarborough, and L. Yeykelis, "Increasing energy efficiency with entertainment media: An experimental and field test of the influence of a social game on performance of energy behaviors," Environment and Behavior, 2013.
[17] J. Nielsen, A. Rodriguez, A. Broussel, S. Flake, G. Sinne, and P. Avila, "Game solution for customer empowerment using water consumption data," 2015, accessed: 10 May 2017. [Online]. Available: http://urbanwater-ict.eu/wp-content/uploads/2015/ 12/UrbanWater-D5.6_v.F.pdf

[18] D. Foster, S. Lawson, M. A. Blythe, and P. Cairns, "Wattsup? motivating reductions in domestic energy consumption using social networks," in In Proceedings of the 6th Nordic Conference on Human-Computer Interaction. Extending Boundaries., 2010.

[19] V. D. Luca and R. Castri, "The social power game: A smart application for sharing energy-saving behaviours in the city," in FSEA 2014, 2014, p. 27.

[20] D. Wemyss, R. Castri, V. De Luca, F. Cellina, V. Frick, E. Lobsiger-Kägi, P. G. Bianchi, C. Hertach, T. Kuehn, and V. Carabias, "Keeping up with the joneses: examining community-level collaborative and competitive game mechanics to enhance household electricity-saving behaviour," in Proceedings of the 4th European Conference on Behaviour and Energy Efficiency Behave 2016, 2016.

[21] A. Kamilaris, A. Pitsillides, C. Fidas, and S. Kondepudi, "Social electricity: The evolution of a large-scale, green ict social application through two case studies in cyprus and singapore," In Proc. of ICT for Sustainability (ICT4S), Copenhagen, Denmark, 2015.

[22] D. Casado-Mansilla, I. Moschos, O. Kamara-Esteban, A. Tsolakis, C. E. Borges, S. Krinidis, D. López-de Ipiña, and D. Tzovaras, GreenSoul: An IoT Platform for Empowering Users' Energy Efficiency in Public Buildings. Cham: Springer International Publishing, 2017, pp. 703-714. [Online]. Available: https://doi.org/10.1007/978-3-319-67585-5_68

[23] D. Kotsopoulos, C. Bardaki, S. Lounis, P. G. Thanasis, and K. Pramatari, "Designing an iot-enabled gamification application for energy conservation at the workplace: Exploring personal and contextual characteristics," in Proceedings-30th Bled e-Conference: Digital Transformation-From Connecting Things to Transforming our Lives, 2017.

[24] G. Mylonas, D. Amaxilatis, H. Leligou, T. Zahariadis, E. Zacharioudakis, J. Hofstaetter, A. Friedl, F. Paganelli, G. Cuffaro, and J. Lerch, "Addressing behavioral change towards energy efficiency in european educational buildings," in Global Internet of Things Summit (GIoTS), 2017. IEEE, 2017, pp. 1-6.

[25] A. E. Rizzoli, A. Castelletti, A. Cominola, P. Fraternali, A. D. dos Santos, Storni, Wissmann-Alvese, Bertocchi, J. Novak, and I. Micheel, "The smarth2o project and the role of social computing in promoting efficient residential water use: a first analysis," 7th International Congress on Environmental Modelling and Software, 2014.

[26] M. Z. Huber and L. M. Hilty, "Gamification and sustainable consumption: overcoming the limitations of persuasive technologies," in ICT Innovations for Sustainability. Springer, 2015, pp. 367-385.

[27] B. J. Fogg., "A behavior model for persuasive design," in Proceedings of the 4th International Conference on Persuasive Technology, no. 40, 2009.

[28] V. Tiefenbeck, L. Goette, K. Degen, V. Tasic, E. Fleisch, R. Lalive, and T. Staake, "Overcoming salience bias: How real-time feedback fosters resource conservation," Management Science, vol. 0, no. 0, p. null, 0. [Online]. Available: https://doi.org/10.1287/mnsc.2016.2646

[29] S. Zikos, A. Tsolakis, D. Meskos, A. Tryferidis, and D. Tzovaras, "Conditional random fields-based approach for real-time building occupancy estimation with multi-sensory networks," Automation in Construction, vol. 68 , pp. 128-145, 2016.

[30] D. Piga, A. Cominola, M. Giuliani, A. Castelletti, and A. E. Rizzoli, "Sparse optimization for automated energy end use disaggregation," IEEE Transactions on Control Systems Technology, vol. 24, no. 3, pp. 1044-1051, 2016.

[31] J. Cigler, S. Prívara, Z. Váňa, E. Žáčeková, and L. Ferkl, “Optimization of predicted mean vote index within model predictive control framework: Computationally tractable solution," Energy and Buildings, vol. 52, pp. $39-49,2012$.

[32] S. Fotios, "A revised kruithof graph based on empirical data," LEUKOS, vol. 13 , no. 1, pp. 3-17, 2017.

[33] J. O. Prochaska and W. F. Velicer, "The transtheoretical model of health behavior change," American journal of health promotion, vol. 12, no. 1 , pp. 38-48, 1997.

[34] G. Lohmann, G. Heilmann, U. Hacke, and S. Robinson, "The ICT PSP methodology for energy saving measurement," 2011, accessed: 25 February 2017. [Online]. Available: http://cordis.europa.eu/docs/projects/cnect/6/250496/ 080/deliverables/001-ARES975520CIPCommondeliverableeSESH.pdf 